\title{
Chromosome-scale assembly of the Kandelia obovata genome
}

\author{
Min-Jie Hu', Wei-Hong Sun ${ }^{2,3}$, Wen-Chieh Tsai ${ }^{4}$, Shuang Xiang ${ }^{2,3}$, Xing-Kai Lai ${ }^{5}$, De-Qiang Chen ${ }^{2,3}$, Xue-Die Liư ${ }^{2}$, \\ Yi-Fan Wang ${ }^{2}$, Yi-Xun Le', Si-Ming Chen ${ }^{2,6}$, Di-Yang Zhang $\mathbb{1}^{3}$, Xia Yu ${ }^{3}$, Wen-Qi Hu ${ }^{3}$, Zhuang Zhou ${ }^{3}$, Yan-Qiong Chen ${ }^{3}$, \\ Shuang-Quan Zou ${ }^{2,3}$ and Zhong-Jian Liu'
}

\begin{abstract}
The mangrove Kandelia obovata (Rhizophoraceae) is an important coastal shelterbelt and landscape tree distributed in tropical and subtropical areas across East Asia and Southeast Asia. Herein, a chromosome-level reference genome of K. obovata based on PacBio, Illumina, and $\mathrm{Hi}-\mathrm{C}$ data is reported. The high-quality assembled genome size is $177.99 \mathrm{Mb}$, with a contig N50 value of $5.74 \mathrm{Mb}$. A large number of contracted gene families and a small number of expanded gene families, as well as a small number of repeated sequences, may account for the small K. obovata genome. We found that K. obovata experienced two whole-genome polyploidization events: one whole-genome duplication shared with other Rhizophoreae and one shared with most eudicots ( $\gamma$ event). We confidently annotated 19,138 protein-coding genes in K. obovata and identified the MADS-box gene class and the RPW8 gene class, which might be related to flowering and resistance to powdery mildew in K. obovata and Rhizophora apiculata, respectively. The reference $K$. obovata genome described here will be very useful for further molecular elucidation of various traits, the breeding of this coastal shelterbelt species, and evolutionary studies with related taxa.
\end{abstract}

\section{Introduction}

Mangrove forests are coastal ecosystems with unique biodiversity that provides many ecosystem services and functions ${ }^{1}$. Mangrove loss will increase the threat of coastal hazards (i.e., erosion, storm surges, and tsunamis) to human safety and shoreline development ${ }^{2}$. Specifically, this will reduce coastal water quality and biodiversity and threaten adjacent coastal habitats, thereby weakening the main resources on which the human community relies, including a large number of products and services provided by mangroves ${ }^{3,4}$. Therefore, detailed studies and analyses of the genome and evolution of mangroves are

\footnotetext{
Correspondence: Shuang-Quan Zou (zou@fafu.edu.cn) or

Zhong-Jian Liu (zjliu@fafu.edu.cn)

${ }^{1}$ Key Laboratory of Humid Sub-tropical Eco-Geographical Processes of the Ministry of Education, Fujian Normal University, Fuzhou 350007, China

${ }^{2}$ Fujian Colleges and Universities Engineering Research Institute of Conservation and Utilization of Natural Bioresources, College of Forestry, Fujian Agriculture and Forestry University, Fuzhou 350002, China Full list of author information is available at the end of the article. These authors contributed equally: Min-Jie Hu, Wei-Hong Sun
}

urgently required, especially in the context of frequent human disturbance and inevitable sea-level rise.

The mangrove species Kandelia obovata belongs to Rhizophoraceae, which is called "Qiuqie" in Chinese, with the Latin name of $K$. candel in "Flora Reipublicae Popularis Sinicae" ${ }^{\prime 5}$. Later, in 2008, its Latin name was changed to $K$. obovata in the "Flora of China". K. obovata is a woody plant predominantly found in tropical and subtropical tidal salt wetlands distributed from East Asia to Southeast Asia ${ }^{7}$. K. obovata adapts to transitional ecosystems where the land and ocean meet by overcoming periodic and aperiodic tidal effects, which induce high salinity, severe erosion, and anaerobic conditions ${ }^{8}$. $K$. obovata plays a crucial role in protecting biodiversity and combating erosion ${ }^{9,10}$. Specifically, the mangrove $K$. obovata can protect the embankment, accelerate the natural deposition of the beach, filter organic matter and pollutants from inland areas, and provide an ideal habitat for the marine flora and fauna ${ }^{11}$. At the same time, due to its beautiful shape, unique floral pattern and fragrance,

\section{(c) The Author(s) 2020}

(c) (i) Open Access This article is licensed under a Creative Commons Attribution 4.0 International License, which permits use, sharing, adaptation, distribution and reproduction c. in any medium or format, as long as you give appropriate credit to the original author(s) and the source, provide a link to the Creative Commons license, and indicate if changes were made. The images or other third party material in this article are included in the article's Creative Commons license, unless indicated otherwise in a credit line to the material. If material is not included in the article's Creative Commons license and your intended use is not permitted by statutory regulation or exceeds the permitted use, you will need to obtain permission directly from the copyright holder. To view a copy of this license, visit http://creativecommons.org/licenses/by/4.0/. 


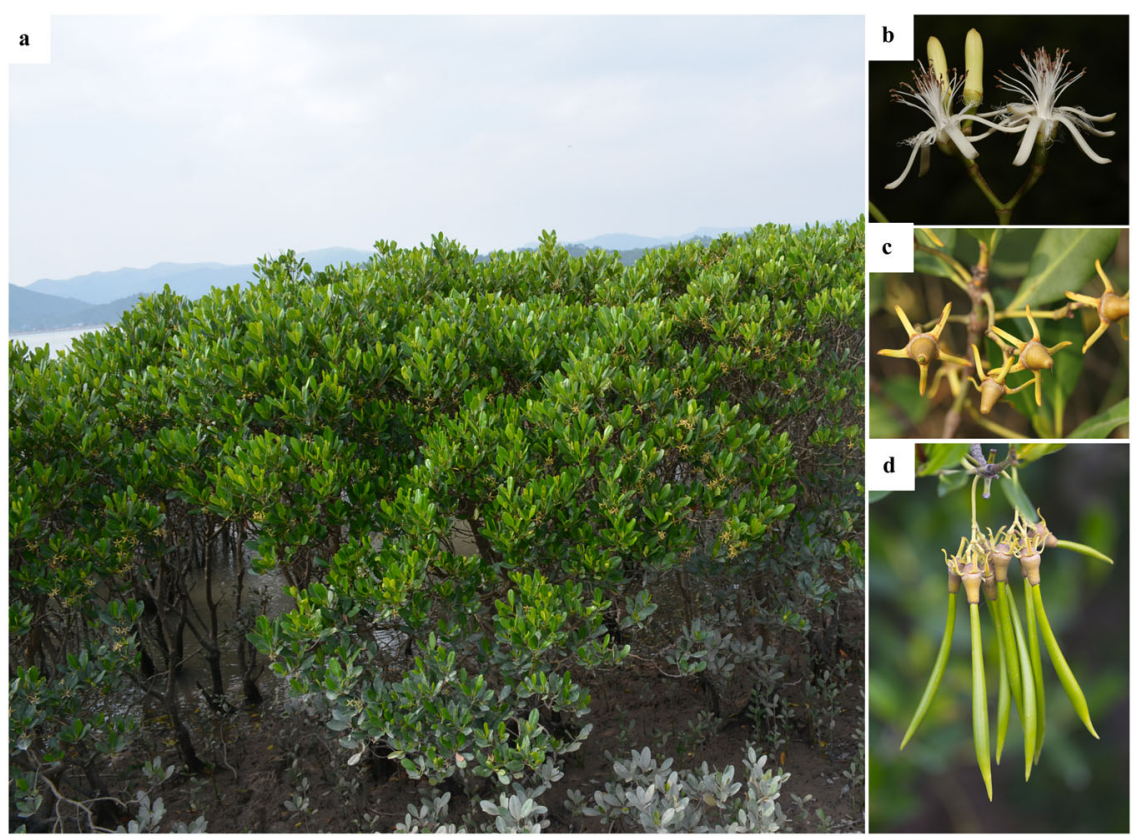

Fig. 1 Morphological features of the flower and fruit of K. obovata. a $K$. obovata trees in a coastal wetland. b Flowers. $\mathbf{c}$ Young fruits. $\mathbf{d}$ Conelike fruits

K. obovata is an excellent coastal wetland landscape plant and horticultural ornamental plant (Fig. 1).

Here, the genome of the mangrove $K$. obovata was sequenced using PacBio sequencing as well as the Illumina next-generation sequencing platform. These data can help clarify the history of mangrove colonization and mangrove adaptation mechanisms in intertidal zones. Furthermore, this study will provide a basis for the conservation of mangrove diversity and in-depth development of genetic resources for mangroves, as well as the development and utilization of coastal horticultural plants.

\section{Results and discussion}

\section{Genome sequence and assembly}

K. obovata contains 36 chromosomes $(2 \mathrm{n}=2 \mathrm{x}=36)^{6}$. To assess genome size, survey sequencing was performed, and $65.27 \mathrm{~Gb}$ of clean data was obtained (Supplementary Table 1). The survey analysis indicated that the K. obovata genome size is $211.86 \mathrm{Mb}$ and has a low level of heterozygosity of approximately $0.38 \%$ (Supplementary Fig. 1). The assembled genome is $178.44 \mathrm{Mb}$ in size, with a scaffold N50 value of $279.55 \mathrm{~kb}$ obtained by using Illumina sequencing (Table 1 ). To improve $K$. obovata assembly quality, we conducted Pacific Biosciences RSII sequencing and obtained $25 \mathrm{~Gb}$ of single-molecule realtime long reads (average read length of $11.9 \mathrm{~kb}$; Supplementary Fig. 2, Supplementary Table 1). The final assembled genome is $177.99 \mathrm{Mb}$ in size, with a contig N50 value of $5.74 \mathrm{Mb}$ (Table 1 ). The quality of the assembly

\section{Table 1 The statistical results of $\mathrm{Hi}-\mathrm{C}$ assembly}

\begin{tabular}{ll}
\hline Assembly & Size (bp) \\
\hline Illumina sequencing assembly & \\
Scaffold N50 & 279,548 \\
Scaffold N90 & 28,239 \\
Longest Scaffold & $1,696,757$ \\
Total Scaffold length & $178,438,058$ \\
PacBio sequencing assembly & \\
Contig N50 & $5,743,053$ \\
Contig N90 & $2,939,642$ \\
Longest Contig & $13,452,090$ \\
Total Contig length & $177,986,124$ \\
BUSCO & $97,3 \%$ \\
Hi-C assembly & \\
Scaffold N50 & $10,026,007$ \\
Scaffold N90 & $7,500,541$ \\
Longest Contig & $13,797,742$ \\
Total Contig length & $178,014,124$ \\
\hline
\end{tabular}

was evaluated using Benchmarking Universal Single-Copy Orthologs (BUSCO $)^{12}$. The results showed that the gene set completeness of the assembled genome is $97.3 \%$, indicating that the $K$. obovata genome assembly is very 
complete and of high quality (Table 1). Finally, highthroughput/resolution chromosome conformation capture (Hi-C) technology was adopted to assess the chromosome-level diploid genome. The results showed that the lengths of the chromosomes ranged from 5.03 to $13.8 \mathrm{Mb}$ (Supplementary Table 2), with a total length of $178.01 \mathrm{Mb}$ and a scaffold $\mathrm{N} 50$ of $10.03 \mathrm{Mb}$ (Fig. 2, Table 1).

\section{Gene prediction and annotation}

We confidently annotated 19,138 protein-coding genes in $K$. obovata (Supplementary Fig. 3, Supplementary Table 3), of which 19,136 (99.17\%) were supported by de novo prediction, transcriptome data, and homolog prediction (Supplementary Table 4). The genome of Rhizophora apiculata, also belonging to Rhizophoreae, has 26,640 protein-coding genes, which is 7502 more than observed in $K$. obovata $^{13}$. The BUSCO $^{12}$ assessment indicated that the completeness of the gene set of the annotated genome was $90 \%$ for K. obovata (Supplementary Table 5). In addition, 105 microRNAs, 307 transfer RNAs, 167 ribosomal RNAs, and 199 small nuclear RNAs were identified in the K. obovata genome (Supplementary Table 6).

Using homology-based and de novo approaches to identify transposable elements (TEs), we estimated that
$24.07 \%$ of the $K$. obovata genome consists of repetitive sequences (Supplementary Figs. 4 and 5 and Supplementary Tables 7 and 8 ) and $29 \%$ of the $R$. apiculata genome consists of repetitive sequences ${ }^{13}$. Compared with those of closely related nonmangrove plant genomes, the repetitive portions of the $R$. apiculata genome, comprising predominantly TE families, are significantly reduced, and the decrease in TE number largely resulted in a general decrease in genome size among true mangroves $^{13}$. The small repetitive sequences may be one reason for the small genome of $K$. obovata. In addition, 18,266 genes were functionally annotated, among which 11,124 and 14,401 were annotated to Gene Ontology terms and Kyoto Encyclopedia of Genes and Genomes terms, respectively, and 12,491 genes were functionally annotated in all five databases (Supplementary Fig. 6, Supplementary Table 9).

\section{Evolution of gene families}

We constructed a phylogenetic tree and estimated the divergence times of $K$. obovata and nine other plant species based on genes extracted from a total of 1095 single-copy families (Supplementary Figs. 7 and 8, Supplementary Table 10). As expected, $K$. obovata was sister to $R$. apiculata (Supplementary Fig. 9). The estimated Rhizophoreae divergence time was 83.15 Mya, and the divergence time between $K$.

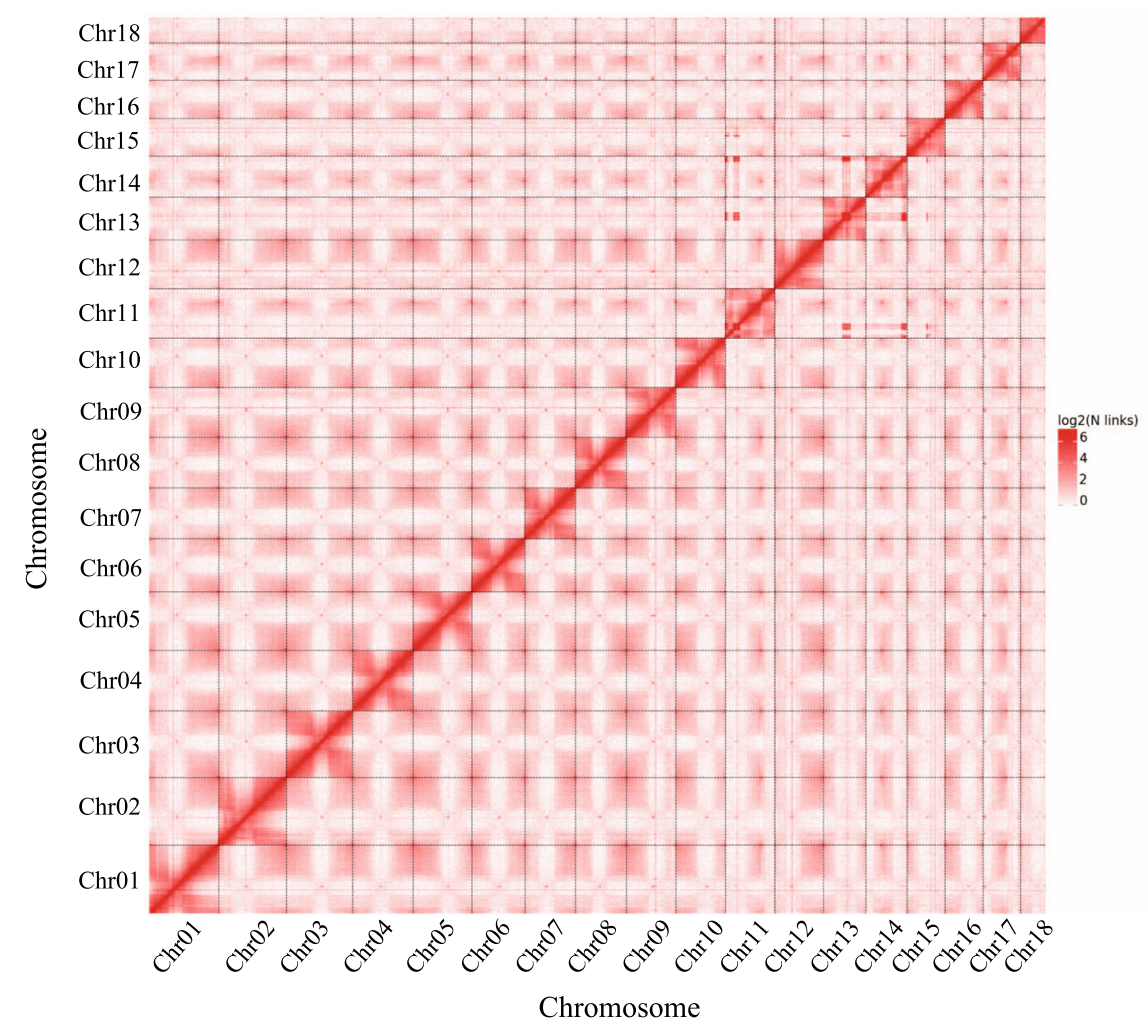

Fig. 2 Intensity signal heat map of the Hi-C chromosome 


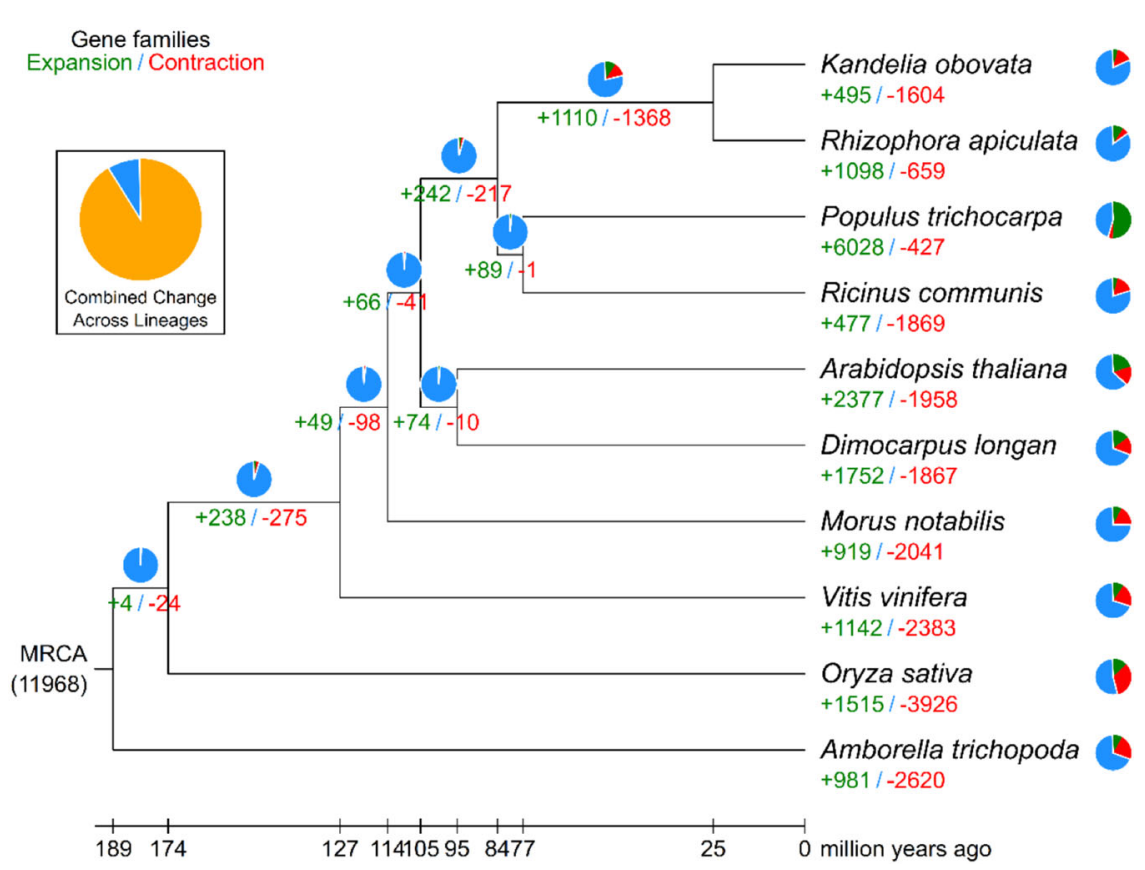

Fig. 3 The expansion and contraction of gene families. The green number indicates the number of expanded gene families, and the red number indicates the number of contracted gene families. The blue color in the circle shows the gene families whose copy numbers are constant, while the orange color represents the proportion of 11,968 gene families in the most recent common ancestor that have expanded or contracted during late differentiation

obovata and R. apiculata was 24.63 Mya (Supplementary Fig. 9). Next, using CAFÉ 3 (ref. ${ }^{14}$ ), we found that 1110 gene families were expanded in the lineage leading to the Rhizophoreae, whereas 1368 families were contracted (Fig. 3). Four hundred and ninety-five gene families were expanded in $K$. obovata, compared with the 1098 in R. apiculata (Fig. 3). At the same time, 1604 gene families were contracted in $K$. obovata, compared with the 659 in $R$. apiculata. K. obovata has more contracted gene families than $R$. apiculata and fewer expanded gene families than $R$. apiculata, which may be the reason that the genome of $K$. obovata is smaller than that of $R$. apiculata. For the expanded gene families, we conducted GO enrichment analysis and found enrichment for the GO terms "structural constituent of cytoskeleton" and "structural constituent of ribosome" (Supplementary Table 11). For the contracted gene families, enrichment was detected for the GO terms "protein kinase activity", "terpene synthase activity", "oxidoreductase activity", "nutrient reservoir activity", "defense response", and "sulfotransferase activity" (Supplementary Table 12). Gene families with K. obovataspecific expansion and contraction might relate to adaptation to $K$. obovata-specific coastal niches. Further research is required to validate the function of these genes.

\section{Synteny analysis and an ancient polyploidization event}

Whole-genome polyploidization events are a feature of many taxa and an efficient mechanisms of genome expansion $^{15}$. To detect the occurrence of polyploidization events in Rhizophoreae, we used the default parameters of JCVI v0.9.14 (ref. ${ }^{16}$ ) to analyze the protein sequences of $K$. obovata, $R$. apiculata, and Vitis vinifera and obtained the gene pairs in the collinear regions. The results showed that there were 11,010 collinear gene pairs between $K$. obovata and R. apiculata, 10,893 collinear gene pairs between $K$. obovata and $V$. vinifera, 3,840 collinear gene pairs within $K$. obovata and 4,646 collinear gene pairs within $R$. apiculata (Supplementary Table 13).

We estimated the distributions of synonymous substitutions per synonymous site $(K s)$ values to more precisely infer the timing of polyploidization events in the $K$. obovata genome. The distributions of $K \mathrm{~s}$ for paralogous $K$. obovata genes showed two peaks, one at $K \mathrm{~s}=$ 0.38 and the other at $K \mathrm{~s}=1.5-1.9$ (Fig. 4, Supplementary Fig. 10a). The Ks distribution of R. apiculata also had two peaks, one at $K s=0.32$ and the other at $K \mathrm{~s}=1.5-1.9$ (Fig. 4, Supplementary Fig. 10b). The results suggested that $K$ obovata and $R$. apiculata experienced two polyploidization events. To confirm these two polyploidization events, we further analyzed the $K$ s distribution of $K$. obovata and $R$. apiculata and that of $K$. obovata and $V$. vinifera. We observed that the $K$ s distribution of $K$. obovata and $R$. apiculata had one peak, at $K \mathrm{~s}=0.1-0.16$, which was smaller than the first peak in the $K$ s 


\section{Ks distribution}

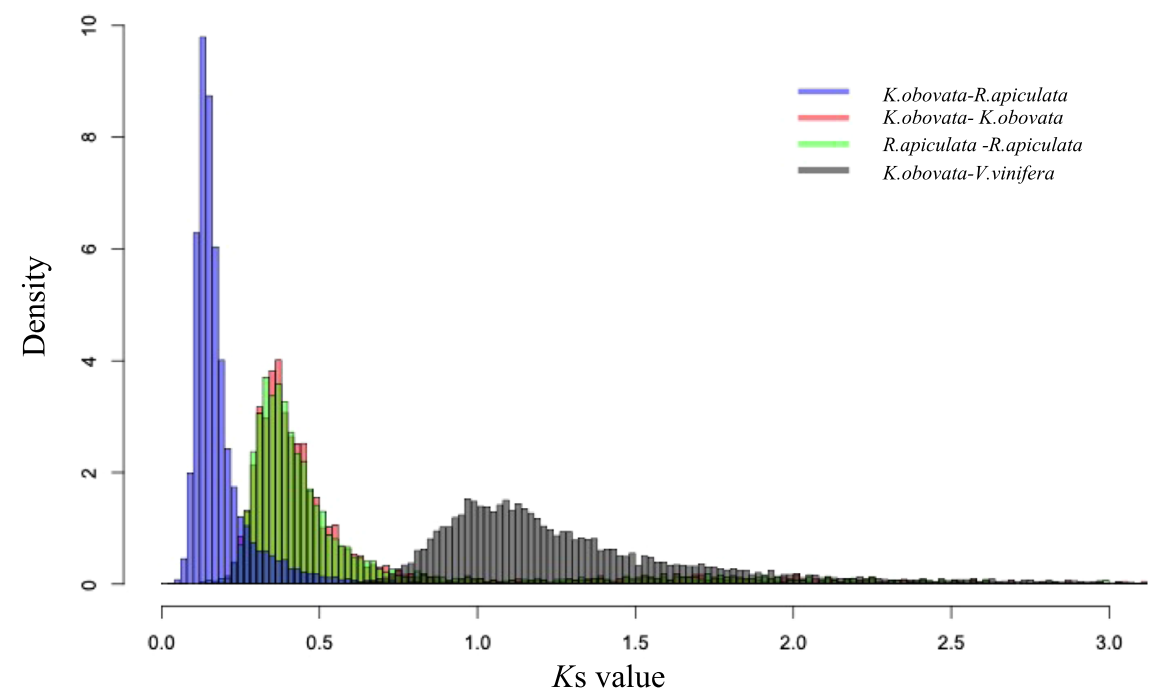

Fig. 4 Ks distributions between $K$. obovata and $R$. apiculata and $K$. obovata and V. vinifera and within $K$. obovata and $R$. apiculata. Peaks of intraspecies Ks distributions indicate ancient whole-genome polyploidization events, and peaks of interspecies Ks distributions indicate speciation events

distributions within $K$. obovata $(K \mathrm{~s}=0.38)$ and $R$. apiculata $(K \mathrm{~s}=0.32)$ (Fig. 4). The first peak in the K. obovata $K s$ distribution $(K \mathrm{~s}=0.38)$ indicates that $K$. obovata shares a whole-genome duplication (WGD) event with other Rhizophoreae. In addition, we found that the $K \mathrm{~s}$ distribution of $K$. obovata and $V$. vinifera had one peak, at $K \mathrm{~s}=0.9-1.4$, which was also smaller than the second peak in the $K \mathrm{~s}$ distributions within $K$. obovata $(K \mathrm{~s}=$ $1.5-1.9)$ and $R$. apiculata (Ks=1.5-1.9) (Fig. 4). The second peak in the $K$. obovata $K \mathrm{~s}$ distribution ( $K \mathrm{~s}=$ 1.5-1.9) indicates that the common ancestor of $K$. obovata and $V$. vinifera experienced an ancient polyploidization event. This event was shared by most eudicots, called the $\gamma$ event, which is an ancient whole-genome triplication event ${ }^{17}$. Finally, we provide direct evidence of gene collinearity, as shown in Fig. 5; the purple peak corresponds to the first peak of the $K$. obovata Ks distribution $(K \mathrm{~s}=0.38)$ and $R$. apiculata $K \mathrm{~s}$ distribution $(K \mathrm{~s}=0.32)$ (Fig. $5 \mathrm{~b}, \mathrm{~d})$, and the green peak corresponds to the second peak of the $K$. obovata Ks distribution ( $K \mathrm{~s}=$ $1.5-1.9)$ and $R$. apiculata $K \mathrm{~s}$ distribution $(K \mathrm{~s}=1.5-1.9)$ (Fig. 5a, c). The purple collinear region is an extra copy of the genomes of K. obovata and R. apiculata, and the green collinear region is also an extra copy of the genes in the genomes of $K$. obovata and $R$. apiculata (Fig. 5). These copies correspond to two polyploidization events of $K$. obovata and $R$. apiculata. Therefore, our study verified that $K$. obovata experienced two polyploidization events: one WGD event shared with Rhizophoreae and one shared with most eudicots ( $\gamma$ event).

\section{MADS-box gene family analysis}

MADS-box genes play a key role in many important processes during plant development, especially during flower development ${ }^{18}$. We evaluated the MADS-box genes in $K$. obovata and $R$. apiculata. The $K$. obovata and $R$. apiculata genomes encode 43 and 65 MADS-box genes, respectively. There are 12 type I and 31 type II MADS-box genes in the $K$. obovata genome and 31 type I and 34 type II genes in the R. apiculata genome (Table 2, Supplementary Table 14). Interactions among type I MADS-box genes promote the initiation of endosperm development ${ }^{19}$. The type I genes of $R$. apiculata were approximately three times more numerous than those of $K$. obovata (Fig. 6a, Table 2). In addition, only 1 pseudogene type I genes were found in the $K$. obovata genome (Supplementary Table 14), suggesting that the type I MADS-box genes of $K$. obovata experienced a lower gain rate and higher loss rate than type II MADS-box genes.

Type II MADS-box genes include two types: MIKC $^{\mathrm{C}}$ and MIKC ${ }^{* 20}$. MIKC $C^{*-t y p e ~ g e n e ~ r e g u l a t i o n ~ h a s ~ a ~ m a j o r ~}$ impact on pollen gene expression ${ }^{21,22}$. Plant MIKC $^{\mathrm{C}}$-type genes are the most widely studied MADS-box genes because they are essential for plant growth and development $^{23,24}$. The $K$. obovata genome has four MIKC"-type genes and $27 \mathrm{MIKC}^{\mathrm{C}}$-type genes, while the $R$. apiculata genome has three MIKC*-type genes and 31 MIKC ${ }^{\mathrm{C}}$-type genes (Fig. 6b, Table 2). Fewer $C / D$-class and $A G L 6$ genes were found in $K$. obovata and $R$. apiculata than in rice, whereas more $B$-AP3-class and $E$-class genes were found in $K$. obovata than in rice (Fig. $6 \mathrm{~b}$ ). $A$-class, $B$-class, 


\section{K. obovata}

a

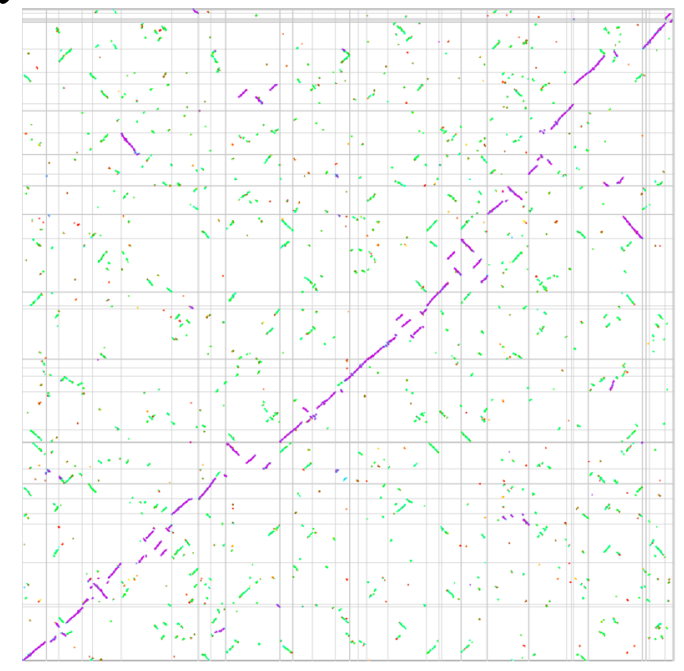

$\mathrm{b}$

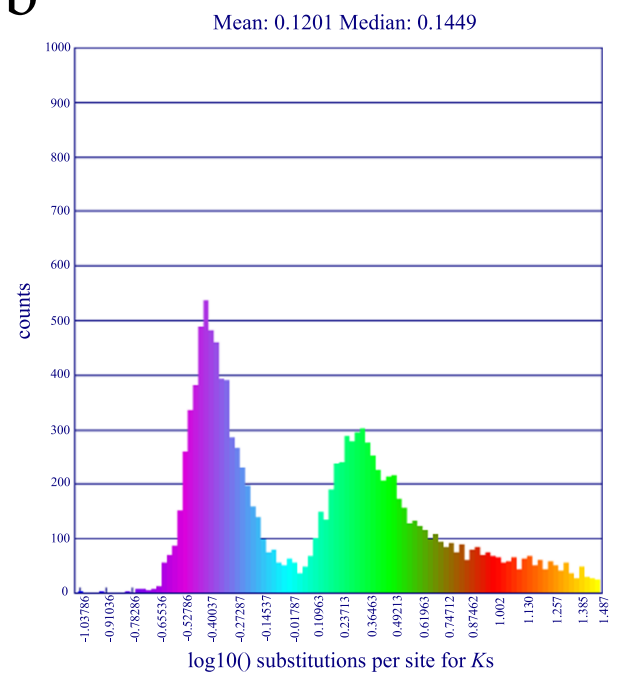

\section{R. apiculata}

C

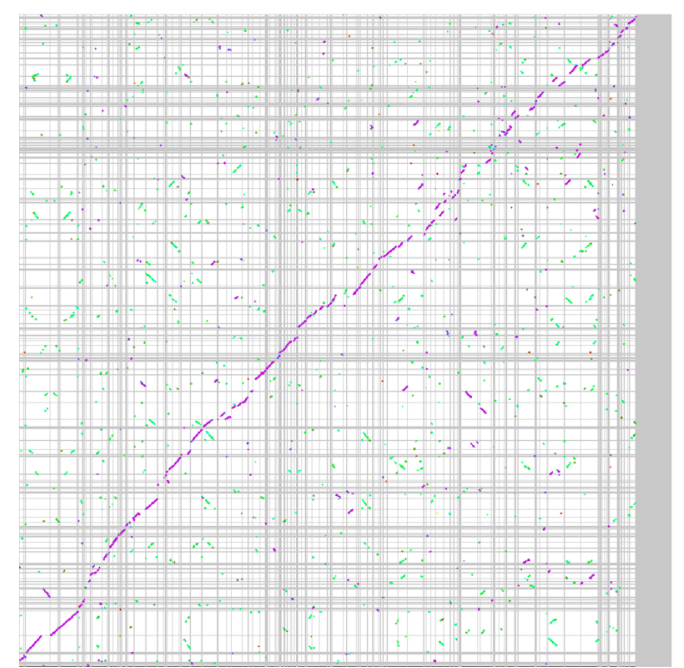

d

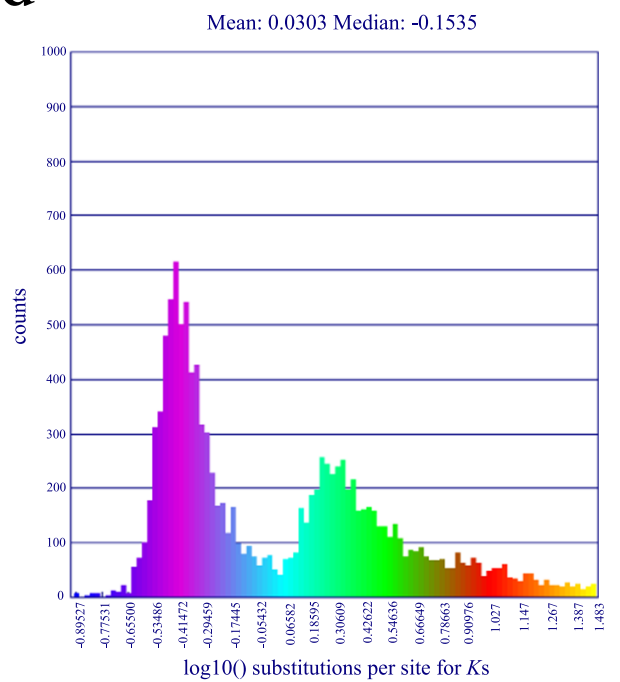

Fig. 5 Collinear point diagram and Ks values corresponding to the collinear blocks. a The collinear point diagram of K. obovata. b Distribution of $\log 10(\mathrm{Ks})$ values of the collinear blocks in K. obovata. $\mathbf{c}$ The collinear point diagram of R. apiculata. $\mathbf{d}$ Distribution of log10 (Ks) values of the collinear blocks in $R$. apiculata. The ordinate of $\mathbf{b}$, $\mathbf{d}$ is the number of gene pairs corresponding to the $K s$ value, and the abscissa is the log 10 (Ks) value

$C / D$-class, and $E$-class gene clades are well known for their roles in the specification of floral organ identity ${ }^{25}$, notably, the ABCDE flowering model ${ }^{26-28}$. K. obovata and $R$. apiculata have the same number of $A$-class and $B$-class genes (five members). K. obovata (six members) has more $E$-class genes than $R$. apiculata (four members), and $R$. apiculata (one member) has fewer $C$-class genes than K. obovata (three members) (Fig. 6b). The AGL12 gene is involved in root cell differentiation ${ }^{29}$, and the ANR1 gene is involved in the regulation of lateral root development ${ }^{30}$. Furthermore, the loss of the AGL12 gene may result in the loss of the ability to develop true roots for terrestrial growth $^{29}$. K. obovata and $R$. apiculata each contain one AGL12-clade gene and one ANR1-clade gene (Fig. 6b), which may be because mangrove roots have adapted to environments at the interface of land and sea. SOC1, SVP, 
Table 2 MADS-box genes in Arabidopsis thaliana, Oryza sativa, Phalaenopsis equestris, K. obovata, and R. apiculata

\begin{tabular}{|c|c|c|c|c|c|}
\hline Category & A. thaliana $a^{\mathrm{a}}$ & O. sativa ${ }^{b}$ & $P$. equestris ${ }^{c}$ & K. obovata & R. apiculata $^{\mathrm{d}}$ \\
\hline Type II (total) & 45 & 44 & 29 & 31 & 34 \\
\hline MIKC & 39 & 39 & 28 & 27 & 31 \\
\hline MIKC $C^{*}$ & 6 & 5 & 1 & 4 & 3 \\
\hline Type I (total) & 61 & 31 & 22 & 12 & 31 \\
\hline $\mathrm{Ma}$ & 25 & 12 & 10 & 6 & 19 \\
\hline$M \beta$ & 20 & 9 & 0 & 1 & 6 \\
\hline MY & 16 & 10 & 12 & 5 & 6 \\
\hline Total & 106 & 75 & 51 & 43 & 65 \\
\hline
\end{tabular}

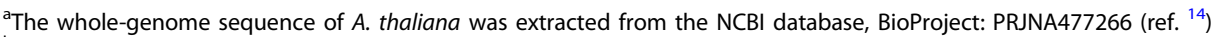

${ }^{\mathrm{b}}$ The whole-genome sequence of $O$. sativa was extracted from rice.plantbiology.msu.edu/

'The whole-genome sequence of $P$. equestris was extracted from the NCBI database, BioProject: PRJNA192198 (ref. ${ }^{15}$ )

${ }^{\mathrm{d}}$ The whole-genome sequence of $R$. apiculata was extracted from http://evolution.sysu.edu.cn/Sequences.html

FLC, and AGL15 regulate flowering time ${ }^{31-34}$. SOC1 integrates multiple flowering signals related to photoperiod, temperature, hormones, and age ${ }^{34}$. Notably, we found that SOC1-like genes were expanded in both $K$. obovata (five members of SOC1) and R. apiculata (seven members of SOC1) (Fig. 6b). Sequence variation among these SOC1-like genes could be associated with the functional diversification of the SOC1 clade in K. obovata and R. apiculata.

\section{Disease resistance-related genes}

Plant resistance genes ( $R$ genes) exist in large families and usually contain a nucleotide-binding site (NBS) domain and a leucine-rich repeat (LRR) domain, denoted $\mathrm{NLR}^{35}$. According to the presence or absence of different domains in the $\mathrm{N}$-terminal region, resistance genes encoding NBS domains can be divided into the TNL (TIR-NBS-LRR), CNL (CC-NBS-LRR), and RNL (RPW8NBS-LRR) groups ${ }^{36}$. A total of 165 and 292 nucleotidebinding site (NBS)-containing $R$ genes were identified in $K$. obovata and $R$. apiculata, respectively; this might be because the distribution of $R$. apiculata is wider than that of $K$. obovata (Fig. 7, Supplementary Table 15).

We selected NLR candidate genes from $K$. obovata and $R$. apiculata with complete domains to construct a phylogenetic tree. The results showed that these candidate genes were divided into the TNL, RNL, and CNL families (Fig. 7). RPW8 is a family of genes with highly specifically expressed characteristics, including resistance to powdery mildew $^{37}$. The phylogenetic tree showed that RPW8 genes were significantly separated from all other CNL genes (Fig. 7). The RPW8 clade contained two K. obovata and three $R$. apiculata genes and clustered with two ADR1 genes from Arabidopsis, indicating that $R P W 8$ genes might be associated with resistance to powdery mildew (Fig. 7).

\section{Conclusion}

Although $K$. obovata is well known as a coastal shelterbelt and landscape tree in tropical and subtropical areas, research on this species has been hampered by a lack of genetic data. We obtained a chromosome-level reference genome of $K$. obovata, assembled a $177.99 \mathrm{Mb}$ genome, and annotated 19,136 protein-coding genes. A large number of contracted gene families and a small number of expanded gene families, as well as a small number of repeated sequences, resulted in a smaller genome in $K$. obovata than in $R$. apiculata. Ks analysis revealed that $K$. obovata experienced two polyploidization events, namely, the recent WGD shared with other Rhizophoreae and the ancient polyploidization event shared with most eudicots ( $\gamma$ event). The Rhizophoreae divergence time was 83.15 Mya, and the divergence time between $K$. obovata and R. apiculata was 24.63 Mya. We identified MADS-box and RPW8 genes in K. obovata, which might be related to flowering and resistance to powdery mildew, respectively. The genomic sequence analysis of the mangrove $K$. obovata helped reveal its mechanisms of adaptation to the intertidal zone; this knowledge is critical for understanding its genetic evolution and reproduction.

\section{Materials and methods \\ DNA preparation and sequencing}

Fresh K. obovata tissues were collected from the Quanzhou Estuary Wetland Provincial Nature Reserve, Fujian Province, China. Genomic DNA was isolated from the fresh leaves of $K$. obovata for de novo sequencing and assembly. Paired-end libraries (500 bp) were constructed according to the Illumina protocol. Genome size and heterozygosity were measured using KmerFreq and GCE based on a $17-K$-mer distribution. In addition, a $20 \mathrm{~kb}$ insert library was constructed according to the PacBio 
a

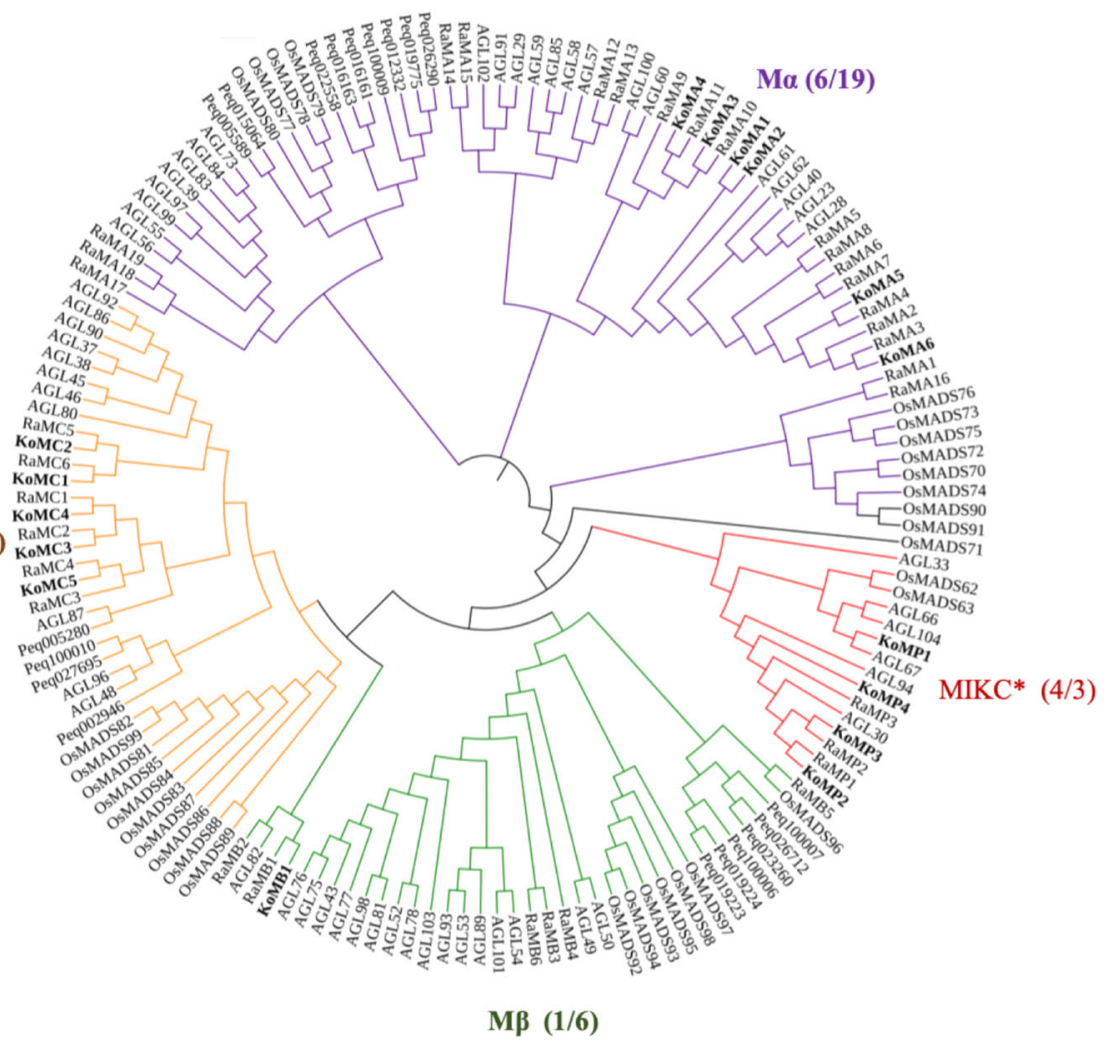

b

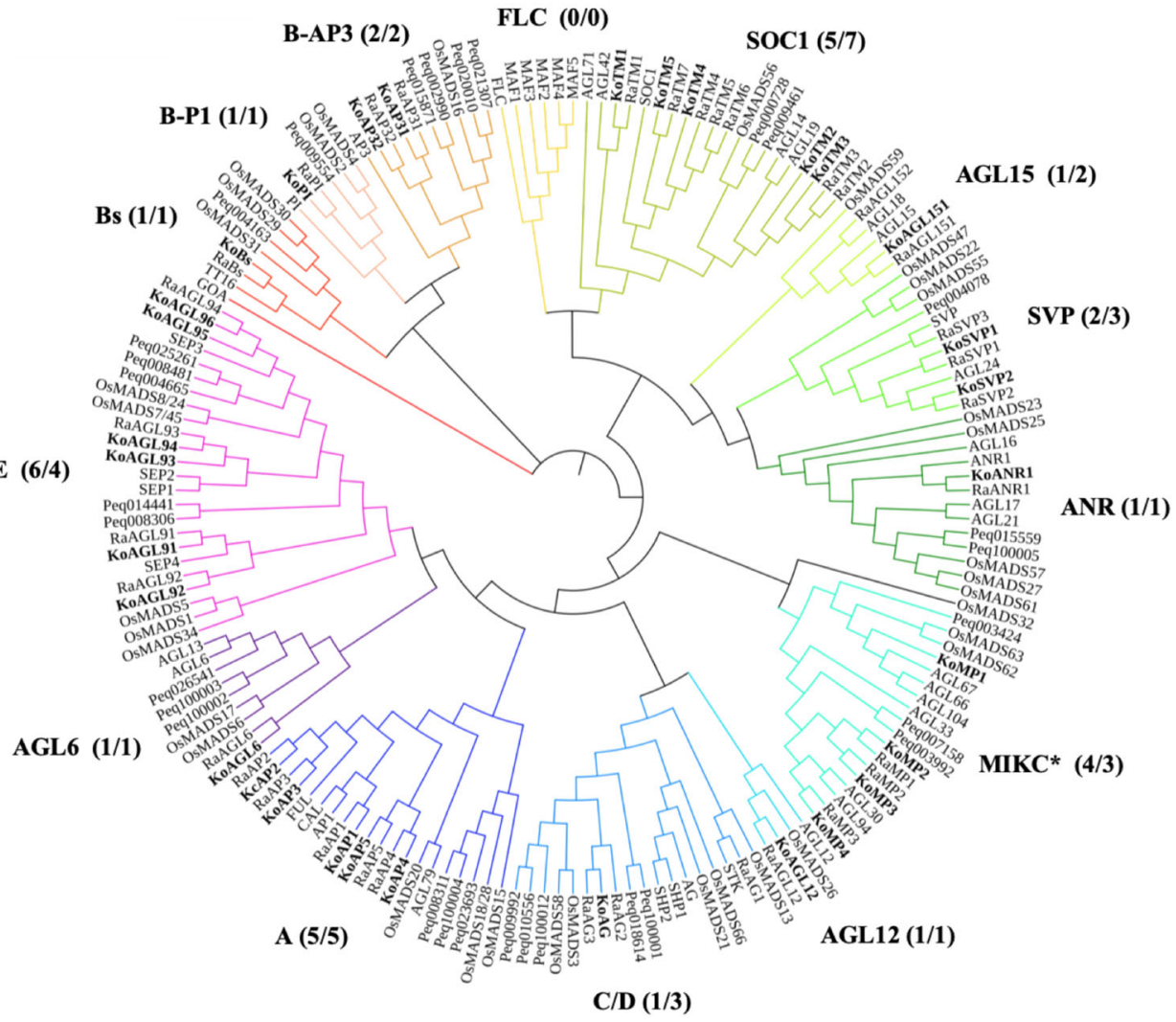

Fig. 6 Phylogenetic analysis of MADS-box genes from A. thaliana, O. sativa, P. equestris, K. obovata, and R. apiculata. a Phylogenetic tree of type I MADS-box genes. $\mathbf{b}$ Phylogenetic tree of type II MADS-box genes. The number on the left in parentheses represents the homologous MADS genes of $K$. obovata, and the number on the right represents the homologous MADS genes of R. apiculata. The bolded gene ID numbers beginning with "Ko" represent the gene IDs of K. obovata; those beginning with "Ra" represent the gene IDs of $R$. apiculata 


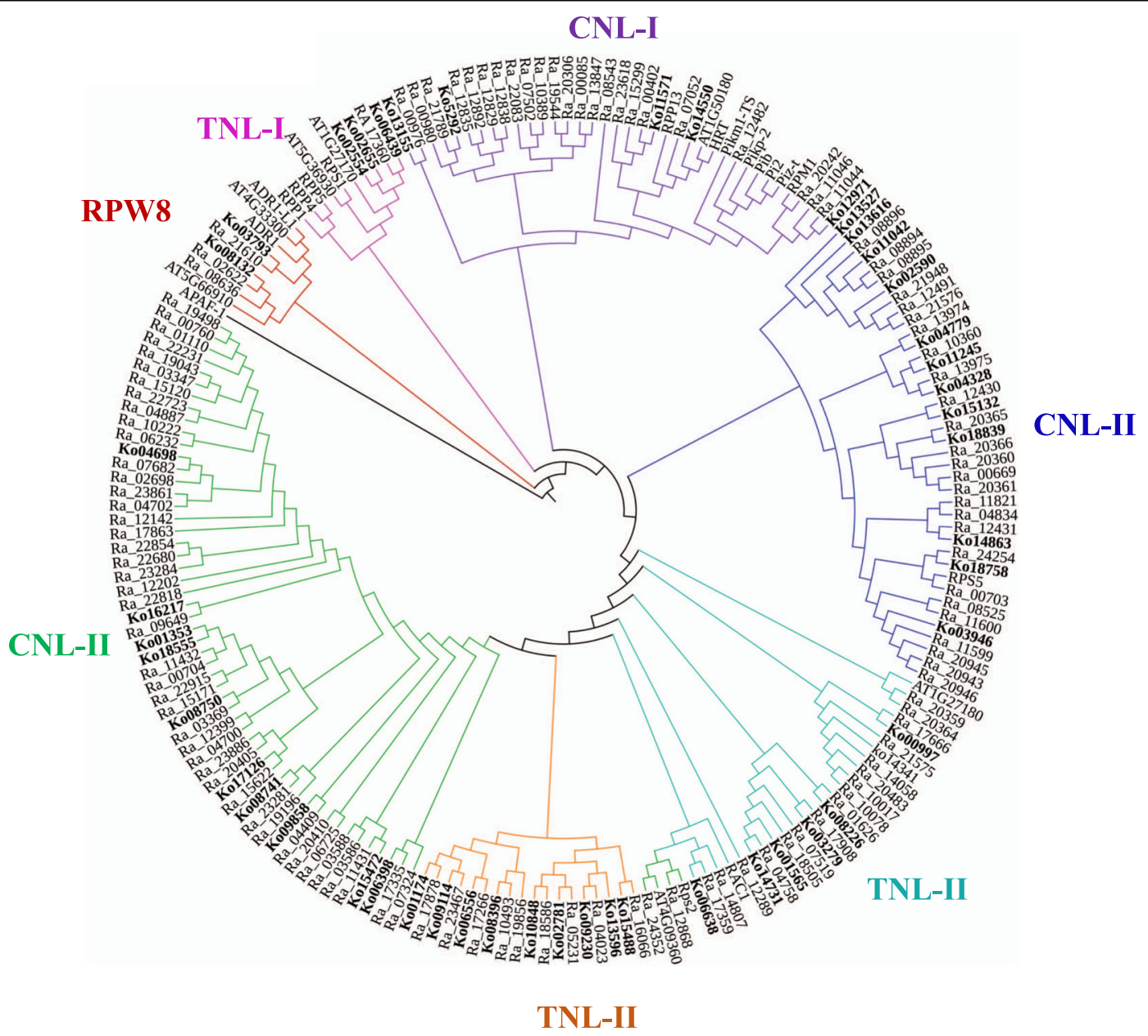

Fig. 7 Phylogenetic reconstruction of the NLR proteins in $K$. obovata and $R$. apiculata. The NBS domain of human apoptotic protease-activating factor-1 (APAF-1) is located at the root of the tree. The bolded gene ID numbers beginning with "Ko" represent the gene IDs of $K$. obovata; those beginning with "Ra" represent the gene IDs of $R$. apiculata

RSII protocol and subsequently sequenced on the PacBio platform (Supplementary Table 1). The transcriptomes of different tissues of $K$. obovata were sequenced on the Illumina platform.

\section{Genome assembly}

De novo assembly of the PacBio reads was performed. FALCON (https://github.com/PacificBiosciences/FALCON) ${ }^{38}$ was used to correct errors in the original data. Then, SMARTdenovo v1.0 was used to assemble the corrected data $^{39}$, and Arrow software (https://github.com/ PacificBiosciences/GenomicConsensus) was used to polish the assembly results. To further eliminate Indel and SNP errors in the assembly sequence, we compared the secondgeneration small-fragment data to the assembly results and corrected the assembly results again with Pilon v1.22 (ref. ${ }^{40}$ ). To confirm the quality of the genome assembly, we performed a BUSCO v3 (ref. ${ }^{12}$ ) (http://busco.ezlab.org/) assessment using single-copy orthologous genes.

\section{$\mathrm{Hi}-\mathrm{C}$ library construction and assembly of the chromosome}

Fresh leaves of $K$. obovata were used to construct a $\mathrm{Hi}-\mathrm{C}$ sequencing library, which was sequenced on the NovaSeq platform. SOAPnuke v1.5.3 (ref. ${ }^{41}$ ) was used to filter the original data (filtration parameter: filter $-\mathrm{n}$ 0.01 -1 20 -q 0.4 -d -M 3 -A 0.3 -Q 2 -i -G --seqType 1) to obtain clean reads. Then, the clean data were compared with the genome using Juicer software ${ }^{42}$. The results were filtered, and misaligned reads were removed. The genome sequence was preliminarily clustered, sequenced, and directed using 3D-DNA ${ }^{43}$. Juicer-box ${ }^{42}$ was again used to adjust, reset, and cluster the genome sequence. Finally, we evaluated genome integrity using BUSCO v3 software ${ }^{12}$. 


\section{Identification of repetitive sequences}

TEs contribute to genome dynamism in terms of both size and structure through insertions and eventual loss ${ }^{44}$. Tandem Repeats Finder (http://tandem.bu.edu/trf/trf. html, v4.07) was used to predict tandem repeats across the genome ${ }^{45}$. TEs were first identified using RepeatMasker v3.3.0 (http://www.repeatmasker.org) and RepeatProteinMask based on Repbase v21.12 (http:// www.girinst.org/repbase $)^{46}$. Then, two de novo prediction software programs, RepeatModeler (http://www. repeatmasker.org/RepeatModeler/) ${ }^{47}$ and LTR_FINDER v1.06 (http://tlife.fudan.edu.cn/ltr_finder/) ${ }^{48}$, were used to identify TEs in the genomes. Finally, repeat sequences with identities $\geq 50 \%$ were grouped into the same classes.

\section{Gene prediction and annotation}

Homology-based, de novo, and transcriptome-based predictions were integrated to predict high-quality proteincoding genes. For homology-based prediction, homologous proteins from five available whole-genome sequences, namely, those of Arabidopsis thaliana, Linum usitatissimum, Populus trichocarpa, Ricinus communis, and Salix purpurea, were aligned to the $K$. obovata genome sequence using Exonerate v2.0 (https://www.ebi.ac.uk/Tools/psa/genewise/ )$^{49}$. Gene structures were generated using GeneWise v2.4.1 $\left(\right.$ ref. ${ }^{50}$ ). Three ab initio prediction software programs, namely, Augustus v3.0.2 (http://bioinf.uni-greifswald.de/ augustus/) ${ }^{51}$, Fgenesh (https://omictools.com/fgeneshtool) ${ }^{52}$, and GlimmerHMM ${ }^{53}$, were employed for de novo gene prediction. Then, the homology-based and ab initio gene structures were merged into a nonredundant gene model using Maker v2.31.8 (ref. ${ }^{54}$ ). TopHat v2.0.11 was used to map RNA-seq reads to the assembly ${ }^{55}$, and Cufflinks v2.2.1 (ref. ${ }^{56}$ ) was applied to combine the mapping results for transcript structural predictions.

The protein sequences of the consensus gene set were aligned to seven protein databases, including GO (The Gene Ontology Consortium) ${ }^{57}$, KEGG (http://www. genome.jp/kegg/) ${ }^{58}$, InterPro (https://www.ebi.ac.uk/ interpro/ $/)^{59}$, Swiss-Prot (http://www.uniprot.org) ${ }^{60}$, and TrEMBL (http://www.uniprot.org// ${ }^{60}$, for predicted gene annotation. The rRNAs were identified by aligning the rRNA template sequences from the $\mathrm{Rfam}^{61}$ database against the genome using the BLASTN algorithm with an E-value cutoff of $1 \mathrm{E}-5$. The tRNAs were predicted using tRNAscanSE v1.3.1 (http://lowelab.ucsc.edu/tRNAscan-SE/) ${ }^{62}$, and other ncRNAs were predicted by Infernal software (http:// infernal.janelia.org/) against the Rfam database.

\section{Phylogenetic analysis}

Genes from whole-genome sequences of ten species (K. obovata, Amborella trichopoda, Arabidopsis thaliana, Dimocarpus longan, Morus notabilis, Populus trichocarpa, Rhizophora apiculata, Ricinus communis, Vitis vinifera, and Oryza sativa) were used for genefamily clustering analysis. OrthoMCL v2.0.9 (ref. ${ }^{63}$ ) was used to identify orthologous groups among the ten species. Pairwise similarities between all protein sequences were calculated using BLASTP with an $E$ value cutoff of $1 \mathrm{E}-5$. To obtain reliable single-copy orthologous groups, we filtered out single-copy orthologous groups containing proteins of length $<200 \mathrm{bp}$. MUSCLE v3.8.31 (ref. ${ }^{64}$ ) was used to perform multisequence alignment of the protein sequences of the filtered single-copy orthologous group, and nucleotide alignment results were obtained by the corresponding relationship between protein sequences and nucleotide sequences. Finally, the nucleotide sequences of the single-copy orthologous group were connected to form a supergene, and then the data set was employed to construct a phylogenetic tree by using the GTR + gamma model in MrBayes ${ }^{65}$.

\section{Estimation of divergence time}

The Markov chain Monte Carlo algorithm for Bayesian estimation was employed to infer the divergence time of each tree node using the MCMCTree module of PAML v4.7 (ref. ${ }^{66}$ ). The nucleic acid replacement model used was the GTR model, and the molecular clock model used was the independent rate model. The MCMC process included 100,000 burn-in iterations and 1,000,000 sampling iterations (with a sample taken every 100 iterations). To obtain a more stable result, the same parameter was executed twice. Calibration times were obtained from TimeTree (http://www.timetree.org).

\section{Gene family expansion and contraction}

We measured the expansion and contraction of orthologous gene families using CAFÉ 3 (https://github.com/ hahnlab/CAFE) ${ }^{14}$. Based on maximum likelihood modeling of gene gain and loss, we analyzed gene families for signs of expansion or contraction using genomic data from the ten species.

\section{Collinearity analysis}

Within collinear segments, genes are conserved in function and sequence and remain highly conserved during the evolution of species. We used the default parameters of JCVI v0.9.14 (https://pypi.org/project/jcvi/) ${ }^{11}$ to analyze the protein sequences of $K$. obovata, $R$. apiculata, and $V$. vinifera and obtained the gene pairs in collinear regions. Then, we used COGE (https://genomevolution. $\mathrm{org} / \operatorname{coge} /$ ) for online analysis, examined the relationship between Ks peaks and collinear regions, and verified the WGD event experienced by the common ancestor of $K$. obovata and $R$. apiculata. 


\section{Whole-genome duplication}

We used Ks distribution analysis to infer WGD events of $K$. obovata and R. apiculata. Diamond v0.9.24 (ref. ${ }^{67}$ ) was used to conduct self-alignment of the protein sequences of the two species and then extract the mutual optimal alignment in the alignment results. Finally, Codeml in the PAML package was used to calculate the $K$ s values $^{39,68}$.

\section{MADS-box analysis}

The hidden Markov model (HMM) profile of the MADSbox gene family (PF00319) was obtained from Pfam (http:// pfam.xfam.org). MADS-box gene family proteins were separately searched with HMMER 3.1 (with the default parameters) ${ }^{69}$. InterProScan v 5.19 (ref. ${ }^{70}$ ) was used to identify MADS-box gene family candidates in the genomes of $K$. obovata and R. apiculata. The genomic data of $R$. apiculata were downloaded from http://evolution.sysu.edu. $\mathrm{cn} /$ Sequences.html. MADS-box gene candidates were further confirmed with the 60 amino acid domains available from SMART ${ }^{71}$ and online BLAST analysis (https://www. ncbi.nlm.nih.gov). Specifically, the protein sequence set for the MADS-box gene candidates was subjected to BLAST analysis against the assembled transcriptomes of the roots, stems, leaves, flowers, and fruits of $K$. obovata with the TBLASTN program. A phylogenetic tree was then constructed using MEGA5 $\left(\right.$ ref. $^{72}$ ) with the default parameters.

\section{Disease resistance genes}

Predicted proteins from the $K$. obovata and $R$. apiculate genomes were scanned using HMMER v3.1 ( $E$-value cutoff of $\left.1 \times 10^{-5}\right)^{69}$ using the HMM corresponding to the Pfam NLR protein family (NB-ARC: PF00931; TIR: PF01582; RPW8: PF05659; LRR: PF00560, PF07723, PF07725 and PF12799). To remove false-positive NBARC domain hits, InterProScan v5.19 was used to check the protein domains of the extracted sequences ${ }^{70}$. The NBS domains of the genes confirmed by both HMMER and InterProScan were extracted according to InterProScan annotation and aligned using MAFFT v7.310 (ref. ${ }^{63}$ ); the alignment was then input into FastTree ${ }^{73}$ with the JTT model and visualized using EvolView ${ }^{74}$.

\begin{abstract}
Acknowledgements
This research was jointly funded by the National Science Foundation of China (41801062), the Special Project for the Cultivation of Major Achievements in the Peak Discipline of Forestry Science (118/71201800709), the Special Subsidy for Leading Talents of Scientific and Technological Innovation in Fujian Province (118/KRC16006A), and the Fujian Forestry Science and Technology Research Project (Min[2019]6). The authors would like to thank Prof. Wenqing Wang (College of the Environment and Ecology, Xiamen University) and Dr. Jiafang Huang (School of Geographical Sciences, Fujian Normal University) for kindly providing the picture of K. obovata.
\end{abstract}

\section{Author details}

${ }^{1}$ Key Laboratory of Humid Sub-tropical Eco-Geographical Processes of the Ministry of Education, Fujian Normal University, Fuzhou 350007, China. ${ }^{2}$ Fujian Colleges and Universities Engineering Research Institute of Conservation and Utilization of Natural Bioresources, College of Forestry, Fujian Agriculture and
Forestry University, Fuzhou 350002, China. ${ }^{3}$ Key Laboratory of National Forestry and Grassland Administration for Orchid Conservation and Utilization at the College of Landscape Architecture, Fujian Agriculture and Forestry University, Fuzhou 350002, China. ${ }^{4}$ Institute of Tropical Plant Sciences and Microbiology, National Cheng Kung University, Tainan 701, China. ${ }^{5}$ Administration of the Quanzhou Bay Estuary Wetland Nature Reserve, Quanzhou 362000, China. ${ }^{6}$ Ocean College, Minjiang University, Fuzhou 350002, China. ${ }^{7}$ Henry Fok College of Biology and Agriculture, Shaoguan University, Shaoguan 512005, China

\section{Data availability}

Genome sequences have been submitted to the National Genomics Data Center (NGDC). PacBio whole-genome sequencing data and Illumina data have been deposited in BioProject/GSA (https://bigd.big.ac.cn/gsa.) ${ }^{75}$ under accession codes PRJCA002330/CRA002395 and the whole-genome assembly and annotation data have been deposited in BioProject/GWH (https://bigd.big ac.cn/gwh) ${ }^{76}$ under accession codes PRJCA002330/GWHACBH00000000.

\section{Conflict of interest}

The authors declare that they have no conflict of interest.

Supplementary Information accompanies this paper at (https://doi.org/ 10.1038/s41438-020-0300-x).

Received: 27 November 2019 Revised: 11 March 2020 Accepted: 16 March 2020

Published online: 02 May 2020

\section{References}

1. Kauffman, J. B. et al. Shrimp ponds lead to massive loss of soil carbon and greenhouse gas emissions in northeastern Brazilian mangroves. Ecol. Evol. 8, 5530-5540 (2018).

2. Gilman, E. L., Ellison, J. C., Duke, N. C. \& Field, C. D. Threats to mangroves from climate change and adaptation options: a review. Aquat. Bot. 89, 237-250 (2008).

3. Nagelkerken, I. et al. The habitat function of mangroves for terrestrial and marine fauna: a review. Aquat. Bot. 89, 155-185 (2008).

4. Walters, B. B. et al. Ethnobiology, socio-economics and management of mangrove forests: a review. Aquat. Bot. 89, 220-236 (2008).

5. Wight et al. in Flora Reipublicae Popularis Sinicae (ed Delectis florae Reipublicae Popularis Sinicae agenda academiae sinicae) Vol. 52, 133-135 (Sciences Press, Beijing, 1983).

6. Qin, H. N. \& David, E. B. in Flora of China (eds Wu, Z. Y., Peter, R.H. \& Hong, D.) Vol. 13, 295-299 (Sciences Press, Beijing, 2009).

7. Sheue, C. R., Liu, H. Y. \& Yong, J. W. H. Kandelia obovata (Rhizophoraceae), a new mangrove species from Asia. Taxon 52, 287-294 (2003).

8. Giri, C. et al. Status and distribution of mangrove forests of the world using earth observation satellite data. Glob. Ecol. Biogeogr. 20, 154-159 (2011).

9. Wardiatno, Y., Mardiansyah, Prartono, T. \& Tsuchiya, M. Possible food sources of macrozoobenthos in the manko mangrove ecosystem, Okinawa (Japan): a stable isotope analysis approach. Trop. Life Sci. Res. 26, 53-65 (2015).

10. Zhou, Q. et al. Characteristics and distribution of microplastics in the coastal mangrove sediments of China. Sci. Total Environ. 31, 134807 (2019).

11. Rogers, A. \& Mumby, P. J. Mangroves reduce the vulnerability of coral reef fisheries to habitat degradation. PLoS Biol. 17, e3000510 (2019).

12. Simao, F. A. et al. BUSCO: assessing genome assembly and annotation completeness with single-copy orthologs. Bioinformatics 31, 3210-3212 (2015).

13. $\mathrm{Xu}, \mathrm{S}$. H. et al. The origin, diversification and adaptation of a major mangrove clade (Rhizophoreae) revealed by whole-genome sequencing. Natl. Sci. Rev. 4, 721-734 (2017)

14. Han, M. V., Thomas, G. W. C., Lugo-Martinez, J. \& Hahn, M. W. Estimating gene gain and loss rates in the presence of error in genome assembly and annotation using CAFE 3. Mol. Biol. Evol. 30, 1987-1997 (2013).

15. McGrath, C. L. \& Lynch, M. Evolutionary significance of whole-genome duplication. in Poly-ploidy and Genome Evolution (eds Soltis, P. S. \& D. E., Soltis) 1-20 (Springer Berlin Heidelberg, Berlin, Heidelberg, 2012).

16. Tang, H. B. et al. JCVI v0.9.14, https://pypi.org/project/jcvi/ (2014).

17. Wu, S. D., Han, B. C. \& Jiao, Y. N. Genetic contribution of Paleopolyploidy to adaptive evolution in angiosperms. Mol. Plant 13, 59-71 (2019). 
18. Zhang, L. et al. Genome-wide identification, characterization of the MADS-box gene family in Chinese jujube and their involvement in flower development. Sci. Rep. 7, 1025 (2017).

19. Masiero, S., Colombo, L., Grini, P. E., Schnittger, A. \& Kater, M. M. The emerging importance of type I MADS box transcription factors for plant reproduction. Plant Cell 23, 865-872 (2011).

20. Henschel, K. et al. Two ancient classes of MIKC-type MADS-box genes are present in the moss physcomitrella patens. Mol. Biol. Evol. 19, 801-804 (2002).

21. Adamczyk, B. J. \& Fernandez, D. E. MIKC* MADS domain heterodimers are required for pollen maturation and tube growth in Arabidopsis. Plant Physiol. 149, 1713-1723 (2009).

22. Liu, Y. et al. Functional conservation of MIKC*-Type MADS box genes in Arabidopsis and rice pollen maturation. Plant Cell 25, 1288-1303 (2013).

23. Theissen, G. \& Melzer, R. Molecular mechanisms underlying origin and diversification of the angiosperm flower. Ann. Bot. 100, 603-609 (2007).

24. Li, C. et al. Genome-wide characterization of the MADS-box gene family in radish (Rahpanus sativus $L$.) and assessment of its roles in flowering and floral organogenesis. Front. Plant Sci. 7, 1390 (2016).

25. Sheng, X. G. et al. Genome wide analysis of MADS-box gene family in Brassica oleracea reveals conservation and variation in flower development. BMC Plant Biol. 19, 106 (2019).

26. Coen, E. S. \& Meyerowita, E. M. The war of the whorls: genetic interactions controlling flower development. Nature 353, 31-37 (1991).

27. Zahn, L. M., Feng, B. \& Ma, H. Beyond the ABC-model: regulation of floral homeotic genes. Adv. Bot. Res. 44, 163-207 (2006).

28. Silva, C. S. et al. Evolution of the plant reproduction master regulators LFY and the MADS transcription factors: the role of protein structure in the evolutionary development of the flower. Front. Plant Sci. 6, 1193 (2015).

29. Ibarra-Laclette, E. et al. Architecture and evolution of a minute plant genome. Nature 498, 94-98 (2013).

30. Zhang, H. \& Forde, B. G. An Arabidopsis MADS box gene that controls nutrient-induced changes in root architecture. Science 279, 407-409 (1998).

31. Searle, I. et al. The transition factor FLC confers a flowering response to vernalization by repressing meristem competence and systemic signaling in Arabidopsis. Genes Dev. 20, 898-912 (2006).

32. Reeves, P. A. et al. Evolution conservation of the FLOWERING LOCUS C mediated vernalization response: evidence from the sugar beet (Bsta vulgaris). Genetics 176, 295-307 (2007).

33. Lee, J. H. et al. Role of SVP in the control of flowering time by ambient temperature in Arabidopsis. Genes Dev. 21, 397-402 (2007).

34. Lee, J. \& Lee, I. Regulation and function of SOC1, a flowering pathway integrator. J. Exp. Bot. 61, 2247-2254 (2010).

35. Lozano, R., Hamblin, M. T., Prochnik, S. \& Jannink, J. L. Identification and distribution of the NBS-LRR gene family in the Cassava genome. BMC Genomics 16, 360 (2015).

36. Xiang, L. X. et al. Genome-wide comparative analysis of NBS-encoding genes in four Gossypium species. BMC Genomics 18, 292 (2017).

37. Xiao, S. et al. The atypical resistance gene, RPW8, recruits components of basal defence for powdery mildew resistance in Arabidopsis. Plant J. 42, 95-110 (2005).

38. Chin, C. S. et al. Phased diploid genome assembly with single-molecule realtime sequencing. Nat. Methods 13, 1050-1054 (2016).

39. Blanc, G. \& Wolfe, K. H. Widespread paleopolyploidy in model plant species inferred from age distributions of duplicate genes. Plant Cell. 16, 1667-1678 (2004).

40. Walker, B. J. et al. Pilon: an integrated tool for comprehensive microbial variant detection and genome assembly improvement. PLOS ONE 9, e112963 (2014).

41. Chen, Y. et al. SOAPnuke: a MapReduce acceleration-supported software for integrated quality control and preprocessing of high-throughput sequencing data. Gigascience 7, 120 (2017).

42. Durand, N. C. et al. Juicer provides a one-click system for analyzing loopresolution Hi-C experiments. Cell Sys 3, 95-98 (2016).

43. Dudchenko, O. et al. De novo assembly of the Aedes aegypti genome using Hi-C yields chromosome-length scaffolds. Science 356, 92-95 (2017).

44. Hawkins, J. S., Proulx, S. R., Rapp, R. A. \& Wendel, J. F. Rapid DNA loss as a counterbalance to genome expansion through retrotransposon proliferation in plants. Proc. Natl Acad. Sci. USA 106, 17811-17816 (2009).

45. Benson, G. Tandem Repeats Finder: a program to analyze DNA sequences. Nucleic Acids Res. 27, 573-580 (1999).
46. Jurka, J. et al. Repbase Update, a database of eukaryotic repetitive elements. Cytogenet. Genome Res. 110, 462-467 (2005).

47. Price, A. L., Jones, N. C. \& Pevzner, P. A. De novo identification of repeat families in large genomes. Bioinformatics 21, 351-358 (2005).

48. Zhao, X. \& Hao, W. LTR_FINDER: an efficient tool for the prediction of fulllength LTR retrotransposons. Nucleic Acids Res. 35, W265-W268 (2007).

49. Slater, G. S. C. \& Birney, E. Automated generation of heuristics for biological sequence comparison. BMC Bioinformatics 6, 31 (2005).

50. Birney, E., Clamp, M. \& Durbin, R. GeneWise and Genomewise. Genome Res. 14, 988-995 (2004).

51. Stanke, M., Schoffmann, O., Morgenstern, B. \& Waack, S. Gene prediction in eukaryotes with a generalized hidden Markov model that uses hints from external sources. BMC Bioinformatics 7, 62 (2006).

52. Grabherr, M. G. et al. Full-length transcriptome assembly from RNA-Seq data without a reference genome. Nat. Biotechnol. 29, 644-652 (2011).

53. Majoros, W. H., Pertea, M. \& Salzberg, S. L. TigrScan and GlimmerHMM: two open source ab initio eukaryotic gene-finders. Bioinformatics 20, 2878-2879 (2004).

54. Holt, C. \& Yandell, M. MAKER2: an annotation pipeline and genome-database management tool for second-generation genome projects. BMC Bioinformatics 12, 491 (2011).

55. Trapnell, C., Pachter, L. \& Salzberg, S. L. TopHat: discovering splice junctions with RNA-Seq. Bioinformatics 25, 1105-1111 (2009).

56. Trapnell, C. et al. Differential gene and transcript expression analysis of RNA-seq. experiments with TopHat and Cufflinks. Nat. Protoc. 7, 562-578 (2012).

57. Ashburner, M. et al. Gene Ontology: tool for the unification of biology. Nat. Genet. 25, 25-29 (2000).

58. Ogata, H. et al. KEGG: Kyoto Encyclopedia of Genes and Genomes. Nucleic Acids Res. 27, 29-34 (1999).

59. Jones, P. et al. InterProScan 5: genome-scale protein function classification. Bioinformatics 30, 1236-1240 (2014).

60. Boeckmann, B. et al. The SWISS-PROT protein knowledgebase and its supplement TrEMBL. Nucleic Acids Res. 31, 365-370 (2003).

61. Griffiths-Jones, S. et al. Rfam: annotating non-coding RNAs in complete genomes. Nucleic Acids Res. 33, D121-D124 (2005).

62. Lowe, T. M. \& Eddy, S. R. tRNAscan-SE: a program for improved detection of transfer RNA genes in genomic sequence. Nucleic Acids Res. 25, 955-964 (1997).

63. Fischer, S. et al. in Current Protocols in Bioinformatics (eds Andreas, D. et al.) Vol. 6, Ch. 6 (Zhang, 2011).

64. Edgar, R. C. MUSCLE: multiple sequence alignment with high accuracy and high throughput. Nucleic Acids Res. 32, 1792-1797 (2004).

65. Castresana, J. Selection of conserved blocks from multiple alignments for their use in phylogenetic analysis. Mol. Biol. Evol. 17, 540-552 (2000).

66. Yang, Z. PAML 4: phylogenetic analysis by maximum likelihood. Mol. Biol. Evol. 24, 1586-1591 (2007)

67. Benjamin, B., Chao, C. \& Daniel, H. H. Fast and sensitive protein alignment using diamond. Nat. Methods 12, 59-60 (2015).

68. Wang, K. et al. The draft genome of a diploid cotton Gossypium raimondii. Nat. Genet. 44, 1098-1103 (2012).

69. Eddy, S. R. Accelerated profile HMM searches. PLoS Comput. Biol. 7, e1002195 (2011).

70. Finn, R. D. et al. InterPro in 2017-beyond protein family and domain annotations. Nucleic Acids Res. 45, D190-D199 (2017).

71. Letunic, I., Doerks, T. \& Bork, P. SMART: recent updates, new developments and status in 2015. Nucleic Acids Res. 43, D257-D260 (2015).

72. Tamura, K. et al. MEGA5: molecular evolutionary genetics analysis using maximum likelihood, evolutionary distance, and maximum parsimony methods. Mol. Biol. Evol. 28, 2731-2739 (2011).

73. Price, M. N., Dehal, P. S. \& Arkin, A. P. FastTree 2-approximately maximumlikelihood trees for large alignments. PLoS ONE 10, e9490 (2010).

74. He, Z. et al. Evolviewv2: an online visualization and management tool for customized and annotated phylogenetic trees. Nucleic Acids Res. 44 W236-W241 (2016).

75. Wang, Y. et al. GSA: Genome Sequence Archive. Genomics Proteomics Bioinformatics 15, 14-18 (2017).

76. Zhang, Z. et al. Database resources of the BIG Data Center in 2019. Nucleic Acids Res. 47, D8-D14 (2019). 